\title{
Aspectos médicos e sociais no atendimento oftalmológico de urgência
}

\author{
Medical and social aspectsofophthalmological emergency care
}

\author{
Newton Kara-Junior ${ }^{(1)}$ \\ Maria Cristina Zanatto ${ }^{(2)}$ \\ Veridiana T.N. Villaça ${ }^{(2)}$ \\ Lúcio Takeshi Nagamati ${ }^{(3)}$ \\ Newton Kara-José ${ }^{(4)}$
}

\begin{tabular}{|c|}
\hline RESUMO \\
\hline $\begin{array}{l}\text { Objetivo: Realizou-se um estudo em cem pacientes no Serviço de } \\
\text { Emergência Oftalmológica do Hospital das Clínicas da Universidade } \\
\text { Estadual de Campinas a fim de analisar as características pessoais e as } \\
\text { dificuldades para obter assistência oftalmológica de forma resolutiva. } \\
\text { Métodos: A amostra apresentou as seguintes características: distâncias } \\
\text { entre } 20 \text { e } 100 \text { quilómetros percorridas por } 50,0 \% \text { dos pacientes a serem } \\
\text { atendidos noHospital das Clínicas da Universidade Estadual deCampinas, } \\
\text { sendo que } 75,0 \% \text { destes necessitaram de acompanhante e } 67,0 \% \text { eram } \\
\text { procedentes de outros municípios. As longas distâncias percorridas } \\
\text { representaram despesas adicionais no tratamento de doenças que muitas } \\
\text { vezes deveriam ser resolvidas localmente. Resultados: Entre os pacientes } \\
\text { encaminhados por oftalmologistas de outros serviços ao Hospital das } \\
\text { Clínicas- Universidade Estadual de Campinas, 87,5\% poderiam ter seu } \\
\text { problema resolvido em nível secundário de atendimento e } 66,7 \% \text { das } \\
\text { urgências verdadeiras e } 60,0 \% \text { das urgências falsas levaram mais de } \\
7 \text { dias para chegar ao ProntoSocorro-UniversidadeEstadualdeCampinas, } \\
\text { sugerindo, nas condições desta pesquisa, uma estruturação precária } \\
\text { dos serviços secundários quanto ao preparo para o atendimento de } \\
\text { urgência e a orientação do paciente. Conclusão: Recomenda-se a } \\
\text { preparação de médicos generalistas e oftalmologistas em participação } \\
\text { resolutiva dos casos de emergência ocular além da instalação de serviços } \\
\text { públicos ou conveniados, secundários e terciários, estrategicamente } \\
\text { distribuídos por todoo Estado deSão Paulo. }\end{array}$ \\
\hline
\end{tabular}

Descritores: Emergência; Oftalmopatias; Acesso aos serviços de saúde.

Trabalho apresentado, sob a forma de tema livre no XXIX Congresso Brasileiro de Oftalmologia Goiânia - 03 a 06 de setembro de 1997 (TL-44).

(1) Pós-graduando da Faculdade de Medicina da Universidade de São Paulo (FMUSP), médico colaborador da Faculdade de Ciências Médicas da Universidade Estadual de Campinas (FCMUNICAMP).

(2) Residente de Oftalmologia do Hospital das Clínicas da Faculdade de Ciências Médicas da Universidade Estadual de Campinas (FCM-UNICAMP).

(3) Acadêmico do sexto ano de Medicina da FCMUNICAMP.

(4) Professor Titular da Disciplina de Oftalmologia da FCM-UNICAMP; Professor Titular de Oftalmologia da Faculdade de Medicina da Universidade de São Paulo (FMUSP).

Endereço para correspondência : Newton Kara Júnior - R. Santa Justina 215/91 - São Paulo (SP) CEP 04545-041

\begin{abstract}
\begin{tabular}{l}
\hline INTRODUÇ̃̃̃O \\
\hline
\end{tabular}
O globo ocular exige especial atenção por sua diferenciação funcional e sua sensibilidade a agressões ${ }^{1}$. Assim, as urgências oftalmológicas representam um perigo iminente de danos oculares, às vezes irreversíveis, devendo ser diagnosticadas e tratadas o mais rápido possível.

De modo geral, a formação médica no que se refere à graduação e pósgraduação no Brasil tende ao enfoque puramente tecnicista, conferindo preparo insuficiente para lidar com fatores sócio-econômicos e culturais da população. Considera-se que o conhecimento médico deve ter características biocêntricas, visando preocupações com o bem-estar físico, psíquico e social do ser humano ${ }^{2}$.

Em pacientes portadores de urgências oculares, a dificuldade de acesso à assistência especializada é particularmente importante pela perda visual que pode ocorrer com o retardo do tratamento. Além das dificuldades para
\end{abstract}


conseguir o primeiro atendimento relacionadas a transporte, disponibilidade de acompanhante e perda do dia de trabalho, o paciente necessita ainda apresentar certas condições para a adesão ao tratamento, mesmo em atendimento gratuito, ou seja, comparecer aos retornos, comprar medicamentos e, às vezes, ser encaminhado a outros serviços ${ }^{2}$.

A literatura nacional pesquisada é escassa quanto à análise das dificuldades enfrentadas por pacientes com urgências oculares na obtenção do tratamento adequado. Em pesquisa com pacientes portadores de glaucoma primário de ângulo aberto, atendidos no Hospital das Clínicas da Universidade Estadual de Campinas (HC-UNICAMP) constataram que $67,4 \%$ não completaram a avaliação oftalmológica solicitada, mostrando as dificuldades dos pacientes em aderir ao tratamento recomendado ${ }^{3}$. Alves et al. ${ }^{4}$, estudando casos de ferimentos perfurantes oculares admitidos no Hospital das Clínicas da Universidade de São Paulo (HC-USP), relataram que $17,4 \%$ dos casos não foram atendidos no primeiro dia após o acidente, evidenciando que mesmo em situações emergenciais, alguns pacientes tardam a buscar assistência oftalmológica.

O presente estudo foi realizado com a finalidade de conhecer características e dificuldades de acesso de pacientes atendidos por urgências oculares no HC- UNICAMP e avaliar a resolubilidade do tratamento prévio.

\section{MÉTODOS}

Foi estudada uma amostra não probabilística formada pelos pacientes que compareceram pela primeira vez no Pronto Socorro da Universidade Estadual de Campinas (PSUNICAMP), nos meses de março e abril de 1997. Não foram considerados os casos de retorno à unidade. Esse serviço funciona diariamente, contando com três residentes de plantão e um docente supervisor, não havendo restrições à procura espontânea.

Foram investigadas as seguintes variáveis: sexo, idade, cidade de procedência, distância aproximada entre a residência e o Hospital das Clínicas, gasto financeiro (em reais) para submeter-se à consulta (englobando todas as despesas do paciente e de seu(s) acompanhante(s) no dia), tempo decorrido entre o sintoma inicial e o primeiro atendimento, tempo decorrido entre o primeiro atendimento (caso tivesse sido realizado em outro serviço) e o atendimento realizado no HC-UNICAMP, diagnóstico, veracidade da urgência, necessidade de encaminhamento de pacientes atendidos previamente em outros serviços e a possibilidade de resolução do caso em nível secundário.

Consideraram-se como urgências verdadeiras os casos que apresentavam risco de diminuição ou perda da visão, a curto e médio prazo, bem como aqueles que requeriam avaliação imediata pela intensidade dos sintomas ${ }^{6}$; a necessidade de encaminhamento foi baseada no diagnóstico estabelecido após o atendimento.
Para a obtenção dessas informações foi aplicado um questionário por entrevista com o próprio paciente ou o acompanhante, pelos residentes do serviço de oftalmologia da Universidade Estadual de Campinas que compunham o grupo de pesquisa.

\section{RESULTADOS}

A amostra foi constituída por 100 indivíduos que procuraram o HC-UNICAMP portadores de urgências oftalmológicas. Apresentaram as seguintes características: $66,0 \%$ eram do sexo masculino; a faixa etária predominante era de vinte a cinqüenta anos (54,0\%), 13,0\% tinham menos de dez anos e $21,0 \%$, mais de 50 anos; $33,0 \%$ procediam da cidade de Campinas, de outras cidades do Estado de São Paulo (61,0\%) e de outros Estados (6,0\%). Observou-se que 50,0\% dos pacientes percorreram distâncias que variaram de 20 a 100 quilômetros para chegar ao PS-UNICAMP e 9,0\% percorreram mais de duzentos quilômetros (Tab. 1).

Dentre os pacientes examinados, 75,0\% necessitaram de acompanhante para comparecer à consulta, sendo este, em $59,0 \%$ dos casos, um membro da família.

Dos 26 pacientes que utilizaram meios de transporte das prefeituras de suas cidades para chegar ao PS-UNICAMP, $76,0 \%$ tiveram gastos totais de até cinco reais no dia da consulta. Porém, dos que vieram por outros meios, 36,4\% gastaram mais de dez reais (Tab. 2).

Em 48,0\% dos casos de urgência, o primeiro atendimento foi realizado por oftalmologistas de outros serviços em $31,0 \%$,

\begin{tabular}{|lc|}
\hline $\begin{array}{c}\text { Tabela 1. Distância entre a moradia do paciente e o Hospital } \\
\text { das Clínicas da Universidade Estadual de Campinas (UNICAMP) }\end{array}$ \\
Distância (Km) & $\mathbf{n = 1 0 0}$ \\
$<20$ & $\%$ \\
$20[-50$ & 29,0 \\
$50[-100$ & 35,0 \\
$100[-200$ & 15,0 \\
200 e mais & 12,0 \\
\hline
\end{tabular}

Tabela 2. Gastos financeiros para obtenção da consulta oftalmológica, conforme o tipo de veículo utilizado para locomoção

\begin{tabular}{|c|c|c|c|c|}
\hline \multirow{3}{*}{ Gasto (Reais) } & \multicolumn{4}{|c|}{ Tipo de veículo } \\
\hline & \multicolumn{2}{|c|}{$\begin{array}{l}\text { Municipal } \\
(n=26)\end{array}$} & \multicolumn{2}{|c|}{$\begin{array}{c}\text { Outro } \\
(n=74)\end{array}$} \\
\hline & f & $\%$ & $f$ & $\%$ \\
\hline $0-5$ & 20 & 77,0 & 24 & 32,5 \\
\hline $6-10$ & 5 & 19,2 & 23 & 31,1 \\
\hline $11-20$ & - & - & 7 & 9,4 \\
\hline \multirow[t]{2}{*}{$>20$} & 1 & 3,8 & 20 & 27,0 \\
\hline & \multicolumn{2}{|c|}{$\bar{x}=R \$ 4,70$} & \multicolumn{2}{|c|}{$\bar{x}=R \$ 24,10$} \\
\hline
\end{tabular}


por oftalmologistas do PS-UNICAMP. Médicos não oftalmologistas foram responsáveis por $19,0 \%$ e $2,0 \%$ dos casos receberam o primeiro atendimento em farmácias. O sistema de saúde mais procurado pelos pacientes para as consultas iniciais foi o SUS $(79,0 \%)$, seguido por atendimentos particulares $(10,0 \%)$, de convênio $(9,0 \%)$ e de farmácias $(2,0 \%)$.

Dos pacientes que foram inicialmente atendidos por oftalmologistas de outros serviços (48 casos), verificou-se que $50,0 \%$ destes poderiam ter sido diagnosticados e tratados adequadamente a nível secundário. Desses pacientes passíveis de serem tratados a nível secundário, 87,5\% foram encaminhados desnecessariamente para centros de referência especializados e os outros procuraram esses serviços por livre iniciativa.

As doenças infecciosas foram as principais causas de atendimento $(34,0 \%)$ seguidas por traumas $(20,0 \%)$ (Tab.3).

As urgências verdadeiras representaram $61,0 \%$ dos casos, em que o tempo decorrido desde o sintoma inicial até o primeiro atendimento foi de até 6 dias em 55,7\% destes casos. Porém, 44,3\% procuraram assistência oftalmológica após 7 dias ou mais. Resultados semelhantes foram observados em relação às falsas urgências: $46,2 \%$ dos pacientes buscaram atendimento depois de 7 dias (Tab. 4). Quanto aos pacientes encaminhados por outros serviços, $66,6 \%$ dos casos de urgências verdadeiras acorreram ao P.S. - UNICAMP após 7

Tabela 3. Diagnósticos estabelecidos no atendimento oftalmológico de urgência

\begin{tabular}{lc} 
& $(\mathbf{n}=\mathbf{1 0 0})$ \\
Diagnóstico & $\%$ \\
Infecção & 34,0 \\
Trauma & 20,0 \\
Tumores & 11,0 \\
Descolamento de retina & 8,0 \\
Uveítes & 7,0 \\
Glaucoma & 6,0 \\
Olho seco & 2,0 \\
Sem alterações patológicas & 3,0 \\
Outros * & 9,0 \\
* Descolamento de vítreo posterior (2), Ectrópio (1), Esotropia congênita (1), \\
catarata senil (1), atrofia óptica (1), microftalmia (2), oclusão de veia central \\
de retina (1). \\
\hline
\end{tabular}

\begin{tabular}{|c|c|c|c|c|}
\hline \multirow{3}{*}{ Tempo (dias) } & \multicolumn{4}{|c|}{ Qualificação da urgência } \\
\hline & \multicolumn{2}{|c|}{$\begin{array}{c}\text { Verdadeiro } \\
(n=61)\end{array}$} & \multicolumn{2}{|c|}{$\begin{array}{l}\text { Falso } \\
(n=39)\end{array}$} \\
\hline & $f$ & $\%$ & $f$ & $\%$ \\
\hline $0-1$ & 20 & 32,7 & 13 & 33,3 \\
\hline $2-6$ & 14 & 22,9 & 8 & 20,5 \\
\hline $7-14$ & 6 & 10,0 & 8 & 20,5 \\
\hline $15-30$ & 10 & 16,4 & 4 & 10,3 \\
\hline \multirow[t]{2}{*}{$>\quad 30$} & 11 & 18,0 & 6 & 15,4 \\
\hline & \multicolumn{2}{|c|}{$\overline{\mathrm{X}}=11,0$ dias } & \multicolumn{2}{|c|}{$\bar{X}=15,5$ dias } \\
\hline
\end{tabular}

dias da realização do encaminhamento, o mesmo ocorrendo com os casos de falsas urgências $(60,0 \%)$ (Tab. 5).

\section{DISCUSSÃO}

A maior proporção de pacientes do sexo masculino $(66,0 \%)$ e de vinte a cinquenta anos $(54,0 \%)$ no atendimento do PS-UNICAMP, provavelmente está relacionada à maior exposição a fatores de risco inerentes a trabalho, trânsito e esporte, ocasionando traumas oculares ${ }^{4-7}$.

A maioria dos pacientes atendidos no HC-UNICAMP $(67,0 \%)$ não residiam em Campinas. Layaun et al. ${ }^{7}$ constataram que $30,0 \%$ dos pacientes acometidos por urgências oculares, que procuraram o HC-USP - Ribeirão Preto, procediam, também de outros municípios. Essa diferença de resultados talvez indique que, no presente estudo, a carência de recursos médicos especializados em regiões próximas a Campinas acarrete aumento da procura ao HC-UNICAMP e/ou a maior área intermunicipal de abrangência deste.

Observou-se que $50 \%$ dos pacientes percorreram distâncias que variaram de 20 a 100 quilômetros para chegar ao PSUNICAMP e $9 \%$ percorreram mais de 200 quilometros. A distância geográfica entre a residência do paciente e o centro de atendimento constitui, em geral, barreira para o acesso ao tratamento, principalmente quando se leva em consideração o inesperado do caso, translado, necessidade de acompanhante, afastamento do trabalho, condições sócio-econômicas insuficientes e necessidade de retorno, que se convertem em causas do retardo do atendimento e do conseqüente prejuízo visual.

$\mathrm{Na}$ ocasião da consulta, a maior parte dos pacientes dispunham de acompanhante que, em geral, era um membro da família. Dos que se utilizaram de transporte gratuito do município de origem ao HC-UNICAMP, grande parte teve gastos totais de até cinco reais no dia da consulta e dos que vieram por outros meios, prevaleceram os que gastaram mais do que dez reais. A esses gastos, soma-se ônus decorrente da perda de horas de trabalho do paciente e do acompanhante além de prejuízo aos orgãos governamentais que custeiam transporte e à sociedade que perde força de trabalho.

\begin{tabular}{|c|c|c|c|c|}
\hline \multirow{3}{*}{ Tempo (dias) } & \multicolumn{4}{|c|}{ Qualificação da urgência } \\
\hline & \multicolumn{2}{|c|}{$\begin{array}{c}\text { Verdadeiro } \\
(n=42)\end{array}$} & \multicolumn{2}{|c|}{$\begin{array}{c}\text { Falso } \\
(n=15)\end{array}$} \\
\hline & $f$ & $\%$ & f & $\%$ \\
\hline $0-1$ & 10 & 23,8 & 5 & 33,3 \\
\hline $2-6$ & 4 & 9,5 & 1 & 6,7 \\
\hline $7-14$ & 6 & 11,9 & 2 & 13,3 \\
\hline $15-30$ & 11 & 27,4 & 1 & 6,7 \\
\hline \multirow[t]{2}{*}{$>30$} & 11 & 27,4 & 6 & 40,0 \\
\hline & \multicolumn{2}{|c|}{$\bar{x}=22,0$ dias } & \multicolumn{2}{|c|}{$\bar{X}=17,5$ dias } \\
\hline
\end{tabular}


Dentre os 69 pacientes que receberam atendimento anterior ao realizado no PS-UNICAMP, a grande maioria $(69,6 \%)$ foi atendida por oftalmologistas, sendo que $50 \%$ desses casos poderiam ser diagnosticados e tratados adequadamente a nível secundário por um especialista bem preparado, utilizando aparelhagem mínima de consultório (lâmpada de fenda, oftalmoscópio direto, tonômetro e retinoscópio). Destes pacientes passíveis de serem tratados a nível secundário, $87,5 \%$ foram encaminhados desnecessariamente para centros de referência especializados e outros vieram por livre iniciativa ou para conferir o tratamento, o que mostra falta de adesão ao primeiro serviço. Apenas 27,5\% haviam sido atendidos inicialmente por médicos não oftalmologistas.

Segundo Edwards ${ }^{8}$, um médico generalista de prontosocorro bem preparado é capaz de resolver $69,0 \%$ dos casos de urgência oftalmológica que recebe e encaminhar corretamente $100,0 \%$ dos casos que não consegue resolver. Admite-se, então, que os pacientes poderiam se beneficiar de medidas de primeiros socorros e orientações quanto às urgências oculares, se atendidos inicialmente por médicos não oftalmologistas bem preparados (provavelmente de mais fácil acesso) até receberem o tratamento definitivo com o oftalmologista, uma vez que muitos hospitais e prontosocorros não dispõem de oftalmologistas de plantão.

O recurso de saúde mais utilizado para os pacientes previamente atendidos foi o SUS $(81,0 \%)$, seguido dos atendimentos particulares $(10,0 \%)$, dos convênios $(9,0 \%)$ e das farmácias (2,0\%), o que sugere baixo poder aquisitivo da população atendida no HC-UNICAMP. Daí a importância da resolubilidade do serviço inicial, levando em consideração que esses pacientes geralmente têm maior dificuldade de se deslocar para outros centros de atendimento, vindo inclusive, em alguns casos, a abandonar o tratamento.

As urgências verdadeiras representaram $61,0 \%$ dos casos, resultado semelhante ao encontrado por Layaun e col. (1990) $(58,5 \%)$ no HC-USP - Ribeirão Preto ${ }^{7}$, mostrando que, em muitos casos, não havia indicação de atendimento imediato, com conseqüente sobrecarga desnecessária do serviço de urgência e causando maior demora no atendimento dos pacientes realmente necessitados ${ }^{9}$. Os traumas e as doenças infecciosas foram as causas prevalentes entre os pacientes atendidos, podendo estar relacionados à faixa etária predominante constituída por adultos em fase produtiva e em maior exposição a fatores de risco ${ }^{7-8}$.

O tempo decorrido do sintoma inicial ao primeiro atendimento foi semelhante para urgências falsas e verdadeiras, sugerindo a falta de informações da população sobre urgências oculares ou incapacidade de vencer "barreiras" no acesso ao atendimento. Entre os pacientes encaminhados por outros serviços, $66,7 \%$ dos casos de urgências verdadeiras e $60,0 \%$ de urgências falsas tardaram mais de 7 dias para obter o atendimento no PS-UNICAMP. Esses fatos sugerem a necessidade do aprimoramento da orientação aos pacientes sobre sua doença ocular e necessidade do tratamento adequado, bem como a facilitação do acesso daqueles ao hospital.
Outros fatores que provavelmente resultam na demora em comparecer ao hospital, também, podem ser as grandes distâncias percorridas pela maioria dos pacientes de sua moradia até o HC-UNICAMP, a dificuldade de translado, a falta de acompanhante, a perda do dia de trabalho e as despesas financeiras.

Os casos de urgência em Oftalmologia são importantes por sua especificidade, freqüência e necessidade de qualidade e rapidez do primeiro atendimento, que é essencial para o prognóstico do caso.

Embora $6,1 \%$ dos pacientes que procuram serviço de urgência em hospital geral o façam devido a queixas oftalmológicas ${ }^{8}$, a literatura nacional é carente de informações sobre as características de acesso da população e qualidade de atendimento em casos de urgência ocular.

Os resultados desta pesquisa sugerem a necessidade da criação de pólos de referência oftalmológicos, maior participação resolutiva dos oftalmologistas nos casos de urgência e formação específica de médicos de serviços de emergência. Propõe-se, também, que, a exemplo da UNICAMP e outros hospitais-escola, sejam ministrados anualmente cursos teórico-práticos de Oftalmologia para alunos de graduação e profissionais da área médica. Deve-se, além disso, pleitear que os acadêmicos de Medicina tenham um estágio de urgência em Oftalmologia durante o internato e que os cursos de especialização incluam em seu conteúdo um programa mínimo da área ${ }^{1,10}$.

\section{CONCLUSÕES}

O estudo mostrou que há um desvirtuamento do serviço em relação ao atendimento de falsas urgências $(39,0 \%)$. Os pacientes percorreram longas distâncias, acarretando perdas econômicas e atraso no tratamento de doenças que deveriam ser resolvidas a nível local, principalmente quando se verifica que $87,5 \%$ dos casos encaminhados por oftalmologistas de outros serviços ao HC-UNICAMP poderiam ter sido resolvidos no nível secundário de atendimento.

O considerável contingente de pacientes que procedem de outras localidades e as longas distâncias por eles percorridas sugerem estruturação precária de serviços secundários.

Há necessidade de melhor preparação de médicos generalistas e oftalmologistas visando à participação mais resolutiva nos casos de emergência ocular além da instalação de serviços públicos ou conveniados secundários e terciários estrategicamente distribuídos por todo o Estado de São Paulo.

\section{SUGESTÕES}

Face aos resultados encontrados e a literatura estudada, sugere-se que os cursos de graduação de Oftalmologia dêem mais ênfase a condutas de primeiros socorros, além de ministrar cursos extracurriculares de reciclagem a médicos generalistas que atendem em pronto-socorro. 
Deve-se, também, incentivar o compromisso dos oftalmologistas para a resolução de casos de urgências oculares.

Salienta-se a necessidade de criação de pólos de referência oftalmológica e o incentivo às campanhas de orientação sobre saúde ocular.

\section{ABSTRACT}

Purpose: A survey was carried out on one hundred patients of the Emergency Service of the Ophthalmology Department of the Hospital das Clinicas of the University of Campinas (UNICAMP), in order to analyze the personal characteristics and the barriers against getting resolving ophthalmologic assistance. Variables, were the following: sex, age, home town, average distance between the place of initial symptoms and first visit to the hospital, time spent between the first examination (if performed in any other service) and the examination performed at the Hospital das Clinicas of University of Campinas, diagnosis, veracity of emergency, need to refer patients previously seen in other services to our Service and possibility of assistance and treatment at a secondary level. Methods: The sample showed the following characteristics: distances between 20 and 100 kilometers covered by 50.0\% of the patients to be seen at University of Campinas. $75.0 \%$ of those patients needed someone to stay with them and $67.0 \%$ came from other municipalities. The long distances covered meant additional expenses for the treatment of diseases which should be treated locally. Results: Among the patients referred to University of Campinas by ophthalmologists of other services, $87.5 \%$ could have their diseases treated at a secondary level of assistance and $66.7 \%$ of real emergencies and $60 \%$ of false emergencies took longer than 7 days to reach the emergency room of University of
Campinas. This shows the poor infrastructure of secondary services regarding excellence of emergency care and education of patients. Conclusions: We recommend education of general physi-cians and ophthalmologists for emergency eye care and also the supply of both secondary and tertiary public services or medicare, strategically setup in the whole state of Sao Paulo.

Keywords: Emergencies; Eye diseases; Health services accessibility.

\section{REFERÊNCIAS}

1. Bonatti JÁ, Yamamoto JH, Warren SHC, Santo RM, Kara-José N. Iatrogenia em trauma perfurante do globo ocular. Rev Bras Oftalmol 1987;46:45-8.

2. Kara-José N, Arieta CEL, Temporini ER, Kang KM, Ambrósio LE. Tratamento cirúrgico da catarata senil. óbices para o paciente. Arq Bras Oftalmol 1996;59:573-7.

3. Gullo RM, Costa VP, Benardi L, Kara-José N. Condições visuais de pacientes glaucomatosos em um hospital universitário. Arq Bras Oftalmol 1996;59: 147-50.

4. Alves MR, Kara-José N, Prado Jr I, Usuba FS, Onclinx TM, Marantes CR Ferimento perfurante ocular: 400 casos admitidos na Clínica Oftalmológica do Hospital das Clínicas da Faculdade de Medicina da Universidade de São Paulo. Arq Bras Oftalmol 1995;58:342-5.

5. Bisol SHDF, Reggi JRA. Traumas oculares: nosologia de 1171 casos. Arq Bras Oftalmol 1995;58:105-12.

6. Kara-Junior N, Oliveira Neto JC, Silva ALB, Schellini AS. Acidentes oculares ocupacionais- ocorrência em Botucatu-SP, no período de 1988 a 1992. Arq Bras Oftalmol 1994;57:389-94.

7. Layaun SED, Schor P, Rodrigues MLV. Perfil da demanda de um serviço de oftalmologia em uma unidade de emergência. Rev Bras Oftalmol 1992;51:171-3.

8. Edwards RS. Ophthalmic urgencies in a district general hospital casualty department. Br J Ophthalmol 1987;71:938-42.

9. Jones NP, Hayward JM, Khaw PT, Claoué CMP, Elkington AR. Function of na ophthalmic: "accident and emergency" department: results of a six month survey. Br Med J 1986;292:188-91.

10. Kara-José N, Alves MR. In Moreira JR, Freitas D, Kruta NS. Trauma ocular. Rio de Janeiro:Cultura Médica; 1997. v.1.

\section{CONGRESSO BRASILEIRO DE CATARATA E CIRURGIA REFRATIVA}

\section{1 a 23 de Junho de 2.001 \\ CENTRO DE CULTURA E CONVENÇÕES DE GOIÂNIA}

REALIZAÇÃO: Sociedade Brasileira de Catarata e Implantes Intraoculares

Sociedade Brasileira de Cirurgia Refrativa

Sociedade Brasileira de Administração em Oftalmologia

SECRETARIA EXECUTIVA: Promove Eventos Especiais

Fone: (0xx62) 215-8200 - Fax: (0xx62) 215-8063

e-mail: promove@terra.com.br 